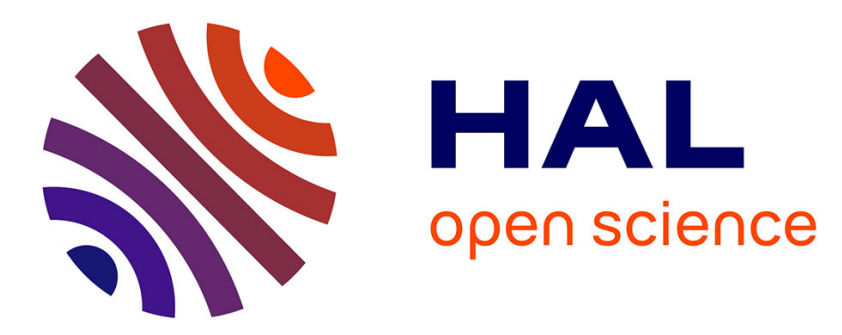

\title{
Active control of the axisymmetric vibration modes of a tom-tom drum
}

Marc Wijnand, Brigitte d'Andréa-Novel, Benoît Fabre, Thomas Hélie, Lionel Rosier, David Roze

\section{- To cite this version:}

Marc Wijnand, Brigitte d'Andréa-Novel, Benoît Fabre, Thomas Hélie, Lionel Rosier, et al.. Active control of the axisymmetric vibration modes of a tom-tom drum. 58th IEEE Conference on Decision and Control, Dec 2019, Nice, France. pp.6887-6892, 10.1109/CDC40024.2019.9029960 . hal-02296092

\section{HAL Id: hal-02296092 \\ https://hal.science/hal-02296092}

Submitted on 2 Sep 2020

HAL is a multi-disciplinary open access archive for the deposit and dissemination of scientific research documents, whether they are published or not. The documents may come from teaching and research institutions in France or abroad, or from public or private research centers.
L'archive ouverte pluridisciplinaire HAL, est destinée au dépôt et à la diffusion de documents scientifiques de niveau recherche, publiés ou non, émanant des établissements d'enseignement et de recherche français ou étrangers, des laboratoires publics ou privés. 


\title{
Active control of the axisymmetric vibration modes of a tom-tom drum
}

\author{
Marc Wijnand, Brigitte d'Andréa-Novel, Benoît Fabre, Thomas Hélie, Lionel Rosier, and David Roze
}

\begin{abstract}
This paper deals with an application of active control of percussion instruments. Our setup consists of a tom-tom drum with a circular membrane, a cylindrical cavity and a circular rigid wall on which a loudspeaker is mounted. The current applied to the loudspeaker is controlled in order to modify the frequencies of the drum membrane modes. First, a PDE model of the axisymmetric transverse vibration of the tom-tom membrane is developed. Subsequently, the equation is recast as an infinite-dimensional port-Hamiltonian system. The port-Hamiltonian framework enables us to develop a numerical scheme that preserves the power balance and guarantees a stable simulation. Finally, a control law for the loudspeaker current is designed to modify the frequency of the first axisymmetric vibration mode of the drum membrane, using finite-time and passivity-based methods.
\end{abstract}

\section{INTRODUCTION}

\section{A. Active control of musical instruments}

Active control of musical instruments consists in adding a control loop to an existing acoustic musical instrument that is being played by a musician. It is an example of active vibration control [1], [2] that enables the musician to enlarge his sound palette while keeping the ergonomics of the original instrument. In addition, it can be used to study the acoustics of the original instrument.

One can distinguish two classes of active control of musical instruments [3], [4], [5], [6], [7]. In acoustical active control, the control acts on a fluid medium. An example is the use of a loudspeaker to create destructive interference in order to cancel sound. In structural acoustical control, the control acts on a solid. Examples include the use of piezoelectric actuators placed on a violin, guitar or percussion instrument.

\section{B. The tom-tom drum}

The tom-tom drum is an unpitched directly struck membrane instrument. It consists of a cylindrical body with a top and bottom membrane and is a standard part of a drum kit.

\footnotetext{
M. Wijnand and B. d'Andréa-Novel are with Sorbonne Université and UMR STMS (Ircam - CNRS - Sorbonne Université), 75014 Paris, France \{marc.wijnand, brigitte. dandreanovel \} @ircam. fr

B. Fabre is with Sorbonne Université and LAM O'Alembert, UMR7190 (Sorbonne Université - CNRS), 75005 Paris, France benoit.fabre@upmc.fr

T. Hélie and D. Roze are with CNRS and UMR STMS (Ircam CNRS - Sorbonne Université), 75014 Paris, France \{thomas . helie, david.roze\} eircam.fr

L. Rosier is with Centre Automatique et Systèmes, Mines ParisTech (PSL Research University), 75014 Paris, France lionel.rosieremines-paristech.fr

M. Wijnand, B. d'Andréa-Novel and L. Rosier are supported by ANR project Finite4SoS (ANR 15 CE23 0007).

M. Wijnand, T. Hélie and D. Roze thank ANR-DFG project Infidhem (ANR 16 CE92 0028).
}

In the field of acoustics of percussion instruments with an air-membrane coupling, instruments such as the tom-tom drum and snare drum [8] and Indian tabla [9], [10] have been considered. Most research is devoted to the timpani or kettledrum [11], [12].

\section{Control type}

The drum membrane is modeled as a partial differential equation (PDE) and the loudspeaker as an ordinary differential equation (ODE). The drum membrane and the loudspeaker are coupled by the air in the cavity volume. We can make following remarks about the control.

1) Distributed (modal) control of a PDE: Depending on the positioning of actuators and sensors, two types of control of PDEs can be defined [13]. In in-domain control, actuators and sensors are located inside the domain. In the case of a lot of distributed actuators and sensors, it is called distributed control. In the alternative case of boundary control, actuators and sensors are located at the boundary of the PDE. In our coupled PDE-ODE system, the PDE is submitted to a distributed pressure that varies as function of a control loop in the ODE.

Furthermore, we opted for a modal control of the PDE. The PDE is projected on eigenfunctions. In the current work, only the first mode is considered.

2) Finite-time control of an ODE: We use finite-time control, a nonlinear control type where the equilibrium point is reached in a finite time [14], which is useful for timeconstrained and robust control [15], [16].

\section{PHYSICAL MODELLING}

\section{A. Layout}

The tom-tom drum coupled to the cavity and loudspeaker is depicted in Figure 1

\section{B. Hypotheses}

The following hypotheses are made in our model.

(H1a) The membrane executes small vertical displacements and its tension is uniform.

(H1b) The vibration of the membrane contains only axisymmetric modes 1 . We suppose that the musician excites the membrane at its center.

(H1c) The sound radiation in air by the membrane is modeled by adding $0.85 a \rho_{\text {air }}$ to the membrane's areal density $\sigma$ [17], $a$ being the membrane's radius and $\rho_{\text {air }}$ the volumetric density of air.

(H2a) The pressure inside the cavity is uniform (lumped

\footnotetext{
${ }^{1}$ We do not consider modes with nodal lines here, for which there is no influence of the cavity.
} 


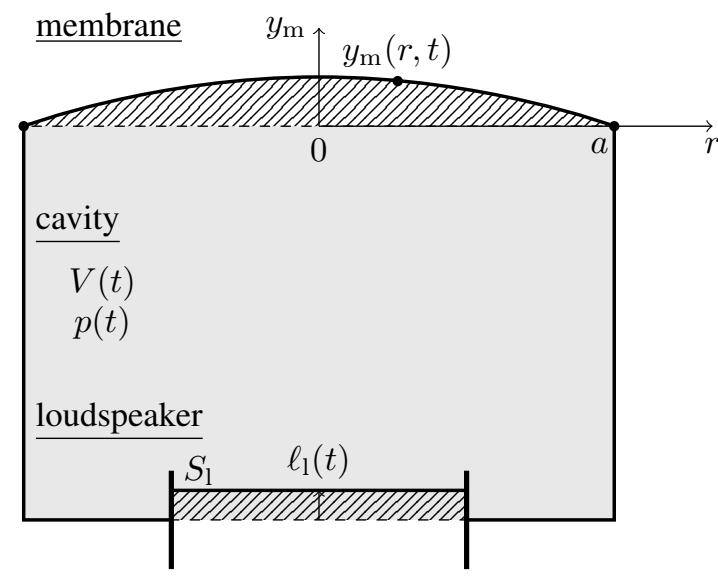

Fig. 1. Geometry of the system

parameter acoustic model without propagation).

(H2b) The air inside the cavity behaves as an ideal gas that is compressed adiabatically.

(H3a) The loudspeaker is modeled as a plane piston.

(H3b) The loudspeaker operates above its resonance frequency.

\section{Physical model}

The model of our system consists of three parts: the membrane (PDE) and the loudspeaker (ODE), that are coupled by the cavity (static relation), as depicted in Figure 1

1) Membrane: Under hypotheses (H1a)-(H1b), one can state the wave equation with a pressure source as [18]

$$
T \boldsymbol{\Delta} y_{\mathrm{m}}(r, t)+p_{c}(t)=\sigma_{\mathrm{m}} \frac{\partial^{2}}{\partial t^{2}} y_{\mathrm{m}}(r, t)+\mu \frac{\partial}{\partial t} y_{\mathrm{m}}(r, t),
$$

where $\boldsymbol{\Delta}=\frac{\partial^{2}}{\partial r^{2}}+\frac{1}{r} \frac{\partial}{\partial r}$ in polar coordinates, $y_{\mathrm{m}}(r, t)$ is the transverse displacement of the membrane, $T$ is the tension $[\mathrm{N} / \mathrm{m}], \sigma_{\mathrm{m}}=\sigma+0.85 a \rho_{\text {air }}$ is the equivalent areal density $\left[\mathrm{kg} / \mathrm{m}^{2}\right]$ according to (H1c), $\mu$ is a friction coefficient $\left[\mathrm{kg} / \mathrm{m}^{2} \mathrm{~s}\right]$ and $p_{c}$ is the net pressure $[\mathrm{Pa}]$ applied to the membrane. The wave velocity in the membrane is $c=\sqrt{T / \sigma_{\mathrm{m}}}$.

The boundary condition is $y_{\mathrm{m}}(a, t)=0$ (membrane clamped at rim). The initial condition representing a stroke with a mallet is $\frac{\partial y}{\partial t}(r, 0)=-V_{0} \mathbb{I}_{\varepsilon} \delta_{0}$, with amplitude $V_{0}$ $\left[\frac{\mathrm{m}}{\mathrm{s}}\right], \mathbb{I}_{\varepsilon}(r)$ a smooth function that varies between a value 1 for $r=0$ and 0 for $r \geq \varepsilon$, and $\delta_{0}$ the Dirac delta function at $t=0$.

2) Cavity: Hypotheses (H2a)-(H2b) correspond to a constant value of $p V^{\gamma}$, with $p$ the total pressure, $V$ the total volume and $\gamma$ the heat capacity ratio. We differentiate this relation and approximate the differentials by differences, using acoustical quantities $p_{\mathrm{c}}$ and $V_{\mathrm{c}}$ that are the deviation values of the instantaneous pressure and volume with respect to the values $p_{0}$ and $V_{0}$ at rest,

$$
\left\{\begin{array}{l}
p_{\mathrm{c}}=p-p_{0} \\
V_{\mathrm{c}}=V-V_{0} .
\end{array}\right.
$$

One obtains the approximate relation (at order 1)

$$
p_{\mathrm{c}} / p_{0}=-\gamma V_{\mathrm{c}} / V_{0} .
$$

The two contributions to the volume change $V_{\mathrm{c}}$ are hatched in Figure 1. There is a volume change due to the transverse movement of the upper membrane and a volume change due to the position of the loudspeaker membrane, which is modeled by a plane piston of vertical position $\ell_{1}$ and effective surface $S_{1}$. Therefore,

$$
V_{\mathrm{c}}(t)=\int_{\mathcal{S}} y_{\mathrm{m}}(r, t) \mathrm{d} \mathcal{S}(r)-S_{\mathrm{l}} \ell_{\mathrm{l}}(t),
$$

with $\mathrm{d} \mathcal{S}(r)=2 \pi r \mathrm{~d} r, r \in[0, a]$. Substituting (3) in (2) yields

$$
p_{c}(t)=-\gamma \frac{p_{0}}{V_{0}}\left[\int_{\mathcal{S}} y_{\mathrm{m}}(r, t) \mathrm{d} \mathcal{S}(r)-S_{\mathrm{l}} \ell_{1}(t)\right] .
$$

3) Loudspeaker: The loudspeaker is modeled as a rigid disc of area $S_{1}$ (H3a). The force balance on this disc yields

$$
m_{1} \ddot{\ell}_{1}(t)+c_{1} \dot{\ell}_{1}(t)+k_{1} \ell_{1}(t)=S_{1} p_{c}(t)+B l i_{1}(t) .
$$

The left-hand side represents the forces of a standard massspring-damper system. The pressure in the cavity applies a force $S_{\mathrm{l}} p_{\mathrm{c}}(t)$ on the loudspeaker. The controlled current $i_{1}(t)$ induces a Laplace force $B i_{1}(t)$, with $B$ the magnetic field $[\mathrm{T}]$ and $l[\mathrm{~m}]$ the length of the loudspeaker coil inside the magnetic field.

\section{Spatial eigenmodes}

Substituting (4) in (1) and setting $\mu=0$ and $\ell_{1}(t)=0$ yields a PDE for the movement of an undamped membrane coupled to the cavity without the controlled loudspeaker:

$$
c^{2} \boldsymbol{\Delta} y_{\mathrm{m}}(r, t)-\frac{\partial^{2} y_{\mathrm{m}}(r, t)}{\partial t^{2}}=\frac{\gamma p_{0}}{\sigma_{\mathrm{m}} V_{0}} \int_{\mathcal{S}} y_{\mathrm{m}}(r, t) \mathrm{d} \mathcal{S}(r) .
$$

In [19], it is shown that for (6), the displacement $y_{\mathrm{m}}(r, t)$ can be written as $y_{\mathrm{m}}(r, t)=\sum_{n=1}^{+\infty} \varphi_{n}(r) z_{n}(t)$ with axisymmetric (under Hypothesis (H1b)) eigenfunctions

$$
\varphi_{n}(r)=w_{n}\left[J_{0}\left(\lambda_{n} r\right)-J_{0}\left(\lambda_{n} a\right)\right],
$$

where $J_{0}(\cdot)$ is a Bessel function of the first kind and $w_{n}$ an arbitrary weight coefficient. It can be shown (see [19]) that the wave number $\lambda_{n}$ satisfies the implicit condition

$$
\lambda_{n}^{2} a^{2} J_{0}\left(\lambda_{n} a\right)=-\frac{\pi a^{4} \gamma p_{0}}{T V_{0}} J_{2}\left(\lambda_{n} a\right) \triangleq-B J_{2}\left(\lambda_{n} a\right),
$$

where the dimensionless group $B$ can be interpreted as the ratio of the restoring force applied by the air in the cavity to the membrane tension [17].

\section{PORT-HAMILTONIAN FORMULATION}

In the Port-Hamiltonian System (PHS, [20], [21]) framework, a system is modeled as consisting of three types of elementary components: energy-storing components, energydissipating components and external components. A PHS model satisfies the power balance. Within this framework, stable simulation [22] and control [23] methods can be developed.

In this section, we recast the system model (1)-(4)-(5) as a PHS. 


\section{A. Definitions}

We use following definitions for (in)finite-dimensional PHSs.

Definition 1 (Finite-dimensional PHS): A classical formulation of a PHS of dimension $n \geq 1$ and state $x \in \mathbb{R}^{n}$ is

$$
\left\{\begin{array}{l}
\dot{\boldsymbol{x}}=(\boldsymbol{J}-\boldsymbol{R}) \boldsymbol{\nabla} H(\boldsymbol{x})+\boldsymbol{G u} \\
\boldsymbol{y}=\boldsymbol{G}^{\boldsymbol{\top}} \boldsymbol{\nabla} H(\boldsymbol{x})
\end{array}\right.
$$

where

- $\boldsymbol{u} \in \mathbb{R}^{q}$ is the input of dimension $q \geq 1$,

- $\boldsymbol{y} \in \mathbb{R}^{q}$ is the output associated with the input (in general different from the measured output $\boldsymbol{y}^{\mathrm{m}}$ ),

- $H(\boldsymbol{x}) \geq 0$ is the Hamiltonian function that represents the total stored energy $E$ of the system as function of its state,

- $\boldsymbol{J}=-\boldsymbol{J}^{\boldsymbol{\top}}$ is an skew-symmetric $(n \times n)$ matrix representing the energy exchanges between energy-storing components,

- $\boldsymbol{R}=\boldsymbol{R}^{\boldsymbol{\top}} \succeq 0$ is a symmetric $(n \times n)$ matrix accounting for the dissipation,

- $\boldsymbol{G}$ is an $(n \times q)$ matrix.

Definition 2 (Infinite-dimensional PHS): An infinite-dimensional PHS is defined as [24], [25]

$$
\left\{\begin{aligned}
\partial_{t} \boldsymbol{x} & =(\mathcal{J}-\mathcal{R}) \delta_{\boldsymbol{x}} \mathcal{H}(\boldsymbol{x})+\mathcal{G} \boldsymbol{u} \\
\boldsymbol{y} & =\mathcal{G}^{*} \delta_{\boldsymbol{x}} \mathcal{H}(\boldsymbol{x})
\end{aligned}\right.
$$

where

- $\mathbb{X}$ is the energy state space,

- $\boldsymbol{u}$ and $\boldsymbol{y}$ are the input and its associated output,

- a scalar product $\langle\cdot, \cdot\rangle_{\mathbb{X}}$ and norm $\|\cdot\|_{\mathbb{X}}$ are defined,

- the Hamiltonian function is obtained as

$$
\mathcal{H}(\boldsymbol{x}) \triangleq \frac{1}{2}\|\boldsymbol{x}\|_{\mathbb{X}}^{2},
$$

- the operator $\delta_{x}$ is the variational derivative [26],

- the operator $\mathcal{J}$ is formally skew-symmetric and the operator $\mathcal{R}$ is non-negative symmetric, w.r.t. the scalar product,

- $\mathcal{G}$ is an operator and $\mathcal{G}^{*}$ its adjoint operator.

\section{B. PHS model}

1) State space: Let $\Omega \triangleq[0, a]$. We define the state as

$$
\boldsymbol{x}=\left[\begin{array}{lllll}
\pi_{\mathrm{m}}(r, t) & \varepsilon_{\mathrm{m}}(r, t) & V_{\mathrm{c}}(t) & \pi_{\mathrm{l}}(t) & \ell_{\mathrm{l}}(t)
\end{array}\right]^{\top},
$$

whose components are respectively the areal momentum of the membrane $\left(=\sigma_{\mathrm{m}} \dot{y}_{\mathrm{m}}\right)$ and its strain $\left(=\nabla y_{\mathrm{m}}\right)$, the incremental volume of the cavity, the loudspeaker membrane's momentum $\left(=m_{1} \dot{\ell}_{1}\right)$ and its vertical position.

The state space is the Hilbert space

$$
\mathbb{X}=H_{0}^{1}\left(\Omega, \mathbb{R}_{\geq 0}\right) \times H^{1}\left(\Omega, \mathbb{R}_{\geq 0}\right) \times \mathbb{R}^{3},
$$

endowed with a scalar product $\langle x, y\rangle_{\mathbb{X}}$ that is defined below according to the total energy of the system.

2) Energy: The total energy of the system is the sum of five contributions.
a) The kinetic energy of the membrane: $\frac{1}{2} \int_{\mathcal{S}} \frac{\pi_{\mathrm{m}}^{2}}{\sigma_{\mathrm{m}}} \mathrm{d} \mathcal{S}(r)$

b) The elastic energy of the membrane:

$$
\frac{1}{2} \int_{\mathcal{S}} T \varepsilon_{\mathrm{m}}^{2} \mathrm{~d} \mathcal{S}(r)
$$

c) The compression energy of the air in the cavity: Starting from $E_{\text {air }}=\int_{0}^{V_{c}}\left(-p_{c}\right) \mathrm{d} V_{c}$ and using the relation (2), one obtains $E_{\text {air }}=\frac{\gamma p_{0} V_{0}}{2}\left(\frac{V_{c}}{V_{0}}\right)^{2} \triangleq \frac{E_{0}}{2}\left(\frac{V_{c}}{V_{0}}\right)^{2}$

d) The kinetic energy of the loudspeaker: $\frac{1}{2} \frac{\pi_{1}^{2}}{m_{1}}$

e) The elastic energy of the loudspeaker spring: $\frac{1}{2} k_{1} \ell_{1}^{2}$

3) Scalar product and Hamiltonian function: We define the scalar product

$$
\begin{array}{r}
\langle\boldsymbol{x}, \boldsymbol{y}\rangle_{\mathbb{X}} \triangleq \int_{\mathcal{S}}\left[\begin{array}{ll}
x_{1}(r) & \left.x_{2}(r)\right]
\end{array}\right] \boldsymbol{L}_{\mathrm{m}}\left[\begin{array}{ll}
y_{1}(r) & y_{2}(r)
\end{array}\right]^{\boldsymbol{\top}} \mathrm{d} \mathcal{S}(r) \\
+\left[\begin{array}{lll}
x_{3} & x_{4} & x_{5}
\end{array}\right] \boldsymbol{L}_{\mathrm{c \& l}}\left[\begin{array}{lll}
x_{3} & x_{4} & x_{5}
\end{array}\right]^{\boldsymbol{\top}}
\end{array}
$$

with

$\boldsymbol{L}_{\mathrm{m}}=\operatorname{diag}\left(\frac{1}{\sigma_{\mathrm{m}}}, T\right) \succ 0, \boldsymbol{L}_{\mathrm{c} \& \mathrm{l}}=\operatorname{diag}\left(\frac{E_{0}}{V_{0}^{2}}, \frac{1}{m_{\mathrm{l}}}, k_{\mathrm{l}}\right) \succ 0$.

With this scalar product, the Hamiltonian function

$$
\mathcal{H}(\boldsymbol{x})=\frac{1}{2}\langle\boldsymbol{x}, \boldsymbol{x}\rangle_{\mathbb{X}}
$$

corresponds to the total energy of the system.

4) Derivative of the Hamiltonian: We are dealing with a mixed PHS, i.e. a combination of a finite-dimensional and an infinite-dimensional PHS. We define a derivative $D_{\boldsymbol{x}} \mathcal{H}(\boldsymbol{x})$ that contains both variational derivatives, for the infinitedimensional state variables, and gradients, for the finitedimensional state variables:

$$
D_{\boldsymbol{x}} \mathcal{H}(\boldsymbol{x})=\left[\frac{\delta_{\boldsymbol{x}_{\mathrm{m}}} \mathcal{H}(\boldsymbol{x})}{\boldsymbol{\nabla}_{\boldsymbol{x}_{\mathrm{c} \& 1}} \mathcal{H}(\boldsymbol{x})}\right]=\mathcal{L} \boldsymbol{x} .
$$

Note that

$$
\mathcal{L} \boldsymbol{x}=\left[\begin{array}{ll|lll}
\frac{\pi_{\mathrm{m}}}{\sigma_{\mathrm{m}}} & T \varepsilon \mid \frac{E_{0}}{V_{0}^{2}} V_{\mathrm{c}} & \frac{\pi_{1}}{m_{1}} & k_{1} \ell_{1}
\end{array}\right]^{\top},
$$

respectively the membrane velocity, the radial projection of the membrane's tension force (for small displacements), the cavity energy density, the loudspeaker velocity and the elastic force on the loudspeaker.

5) PHS: The first equation of the PHS model is (cf. Equation (10) where $\delta_{\boldsymbol{x}}$ is replaced by $D_{\boldsymbol{x}}$ ) is shown in Equation (11) on the next page. Here, $\mathbb{1}$ represents the identity operator.

We note that the operator $\mathcal{J}$ is formally skew-symmetrie ${ }^{2}$ and with domain $\mathcal{D}(\mathcal{J})=H_{0}(\mathrm{grad}) \times H(\operatorname{div}) \times \mathbb{R}^{3}$ where $\operatorname{grad}=\frac{\partial}{\partial r}$ and $\operatorname{div}=\left(\frac{\partial}{\partial r}+\frac{1}{r}\right)$ in polar coordinates.

The output equation of the PHS model corresponds to $\boldsymbol{y}=\mathcal{G}^{\top} \mathcal{L} \boldsymbol{x}=B l \dot{\ell}_{1} \triangleq u_{1}$, the electromotive force. Note that the product between input $i_{1}$ and output $u_{1}$ represents the electrical power supplied to the loudspeaker of the system. This output equation of the PHS will not be considered in the remainder of the paper. 


$$
\begin{aligned}
& \frac{\partial}{\partial t}\left[\begin{array}{c}
\pi_{\mathrm{m}} \\
\frac{\varepsilon_{\mathrm{m}}}{V_{\mathrm{c}}} \\
\hline \pi_{1} \\
\ell_{1}
\end{array}\right]=\left(\left[\begin{array}{cc|c|cc}
0 & \operatorname{div} & -1 & 0 & 0 \\
\operatorname{grad} & 0 & 0 & 0 & 0 \\
\hline \int_{\mathcal{S}} \cdot \mathrm{d} \mathcal{S} & 0 & 0 & -S_{1} & 0 \\
\hline 0 & 0 & S_{1} & 0 & -1 \\
0 & 0 & 0 & 1 & 0
\end{array}\right]-\left[\begin{array}{cc|c|cc}
\mu & 0 & 0 & 0 & 0 \\
0 & 0 & 0 & 0 & 0 \\
\hline 0 & 0 & 0 & 0 & 0 \\
\hline 0 & 0 & 0 & c_{1} & 0 \\
0 & 0 & 0 & 0 & 0
\end{array}\right]\right)\left[\begin{array}{c}
\frac{\pi_{\mathrm{m}}}{\sigma_{\mathrm{m}}} \\
T \varepsilon \\
\hline \frac{E_{0}}{V_{0}^{2} V_{\mathrm{c}}} \\
\hline \frac{\pi_{1}}{m_{1}} \\
k_{1} \ell_{1}
\end{array}\right]+\left[\begin{array}{c}
0 \\
0 \\
\frac{0}{0} \\
\hline B l \\
0
\end{array}\right] i_{1} \\
& \frac{\partial}{\partial \tilde{t}}\left[\begin{array}{c}
\tilde{\pi}_{\mathrm{m}} \\
\tilde{\varepsilon}_{\mathrm{m}} \\
\frac{\tilde{V}_{\mathrm{c}}}{\tilde{\pi}_{1}} \\
\tilde{\ell}_{1}
\end{array}\right]=\left(\left[\begin{array}{cc|c|cc}
0 & \left(\frac{\partial}{\partial \tilde{r}}+\frac{1}{\tilde{r}}\right) & -\alpha \mathbb{1} & 0 & 0 \\
\frac{\partial}{\partial \tilde{r}} & 0 & 0 & 0 & 0 \\
\hline \alpha \int_{\tilde{\mathcal{S}}} \cdot \mathrm{d} \tilde{\mathcal{S}} & 0 & 0 & -\beta_{1} & 0 \\
\hline 0 & 0 & \beta_{1} & 0 & -\beta_{2} \\
0 & 0 & 0 & \beta_{2} & 0
\end{array}\right]-\left[\begin{array}{cc|c|cc}
\xi & 0 & 0 & 0 & 0 \\
0 & 0 & 0 & 0 & 0 \\
\hline 0 & 0 & 0 & 0 & 0 \\
\hline 0 & 0 & 0 & \zeta & 0 \\
0 & 0 & 0 & 0 & 0
\end{array}\right]\right)\left[\begin{array}{c}
\tilde{\pi}_{\mathrm{m}} \\
\tilde{\varepsilon}_{\mathrm{m}} \\
\tilde{V}_{\mathrm{c}} \\
\frac{\tilde{\pi}_{1}}{\tilde{\ell}_{1}}
\end{array}\right]+\left[\begin{array}{c}
0 \\
0 \\
0 \\
\frac{1}{1} \\
0
\end{array}\right]
\end{aligned}
$$

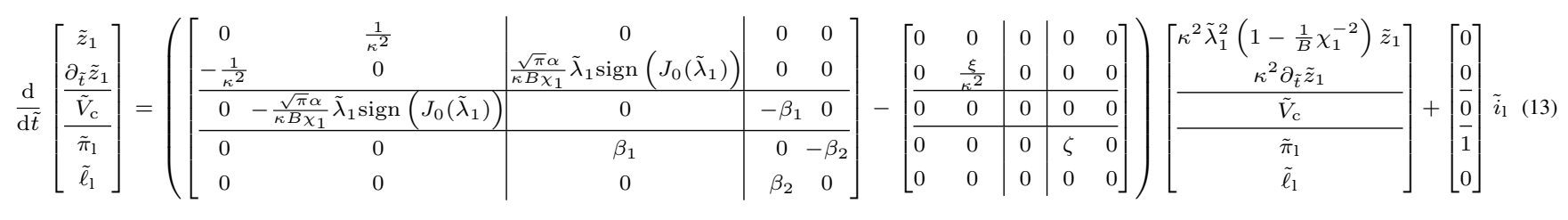

TABLE I

NONDIMENSIONALIZATION OF VARIABLES AND CONSTANTS

$$
\begin{aligned}
& \tilde{r}=\frac{r}{a} \quad \tilde{t}=\frac{c}{a} t \quad \tilde{\mathcal{S}}=\frac{\mathcal{S}}{a^{2}} \\
& \tilde{\pi}_{\mathrm{m}}=\frac{\pi_{\mathrm{m}}}{a^{-1} \sqrt{\sigma_{\mathrm{m}} \gamma p_{0} V_{0}}} \quad \tilde{\varepsilon}_{\mathrm{m}}=\frac{\varepsilon_{\mathrm{m}}}{a^{-1} \sqrt{\gamma p_{0} V_{0} T^{-1}}} \tilde{V}_{\mathrm{c}}=\frac{V_{\mathrm{c}}}{V_{0}} \\
& \tilde{\pi}_{1}=\frac{\pi_{1}}{\sqrt{m_{1} \gamma p_{0} V_{0}}} \quad \tilde{\ell}_{1}=\frac{\ell_{1}}{\sqrt{k_{1}^{-1} \gamma p_{0} V_{0}}} \quad \tilde{\mathcal{H}}=\frac{\mathcal{H}}{\gamma p_{0} V_{0}} \\
& \tilde{i}_{1}=\frac{i_{1}}{c(a B l)^{-1} \sqrt{m_{1} \gamma p_{0} V_{0}}} \quad \zeta=\frac{a c_{1}}{c m_{1}} \quad \xi=\frac{\mu a}{c \sigma_{\mathrm{m}}} \\
& \alpha=\frac{a^{2}}{c} \sqrt{\frac{\gamma p_{0}}{\sigma_{\mathrm{m}} V_{0}}} \quad \beta_{1}=\frac{a S_{1}}{c} \sqrt{\frac{\gamma p_{0}}{m_{1} V_{0}}} \quad \beta_{2}=\frac{a}{c} \sqrt{\frac{k_{1}}{m_{1}}}
\end{aligned}
$$

\section{Dimensionless PHS model}

We nondimensionalize the PHS model in order to simplify subsequent calculations, using the transformations shown in Table I.

We thus obtain the dimensionless PHS shown in Eq. (12) above with $\mathrm{d} \tilde{\mathcal{S}}=2 \pi \tilde{r} \mathrm{~d} \tilde{r}, \tilde{r} \in \tilde{\Omega}=[0,1]$.

\section{MODEL ORDER REDUCTION}

\section{A. Modal projection}

We now project the infinite-dimensional part of the (dimensionless) PHS (12) on a spatial basis and truncate at $N$ modes 3 in order to obtain a finite-dimensional approximative (dimensionless) PHS.

The position of the membrane $\tilde{y}_{\mathrm{m}}$ is thus approximated as a finite sum

$$
\tilde{y}_{\mathrm{m}}(\tilde{r}, \tilde{t}) \approx \sum_{n=1}^{N} \tilde{\varphi}_{n}(\tilde{r}) \tilde{z}_{n}(\tilde{t})
$$

where $\tilde{\varphi}_{n}(\tilde{r})$ are the spatial eigenfunctions of the membrane coupled to the cavity (7) that form an orthonormal basis 4 and $\tilde{z}_{n}(\tilde{t})$ are the corresponding temporal evolutions.

\footnotetext{
${ }^{2}$ This can be shown by integration by parts.

${ }^{3}$ Note that the lowest-order modes are most important for the produced sound 17. Furthermore, these modes are influenced the most by the coupling with the cavity [19].

${ }^{4}$ After definition of a scalar product $\left\langle\tilde{\varphi}_{i}, \tilde{\varphi}_{j}\right\rangle_{\mathrm{P}} \triangleq \int_{\tilde{\mathcal{S}}} \tilde{\varphi}_{i} \tilde{\varphi}_{j} \mathrm{~d} \tilde{\mathcal{S}}(\tilde{r})$ used for the projection, one can prove orthogonality and normalization (given a suited choice of the weights $w_{i}$ ).
}

(Nondimensionalization was done using $\tilde{y}_{\mathrm{m}}=\frac{y_{\mathrm{m}}}{a}, \tilde{\varphi}_{n}=$ $\varphi_{n}$ and $\left.\tilde{z}_{n}=\frac{1}{a} z_{n}.\right)$

\section{B. Port-Hamiltonian approximated model}

The result of this projection is shown in (13) above for the case of $N=1$ mode. (In this formulation, $\kappa \triangleq$ $a \sqrt{T\left(\gamma p_{0} V_{0}\right)^{-1}}$ and $\chi_{1} \triangleq \sqrt{\frac{\tilde{\lambda}_{1}^{4}}{4 B^{2}}-\frac{\tilde{\lambda}_{1}^{2}}{2 B}+\frac{1}{4}+\frac{2}{B}}$.)

\section{PASSIVE FINITE-TIME CONTROL OF THE UNIMODAL MODEL}

\section{A. Control goal and design of the method}

Our control goal is to modify the frequency of the first axisymmetric vibration mode $\left(\tilde{z}_{1}\right)$ of the tom-tom membrane. To this means, we develop a control loop that controls the loudspeaker current $\tilde{i}_{1}$ based on a measurement of the pressure $p_{\mathrm{c}}$ inside the cavity and $\tilde{z}_{1}$, the temporal evolution of the first mode ${ }^{5}$. By changing the vertical position of the loudspeaker, the volume and thus pressure inside the cavity are changed. This pressure acts on the tom-tom membrane and influences its vibrational behavior.

The synthesis of this controller for the loudspeaker current is based on the projected SHP model for $N=1$ mode (13) and consists of 3 steps.

1) The membrane is controlled by a pole placement, the cavity pressure $p_{\mathrm{c}}$ being viewed as an intermediate control input.

2) The cavity couples the three subsystems: the temporal evolution $\tilde{z}_{1}$ of the first membrane mode, the pressure in the cavity $p_{\mathrm{c}}$ and the loudspeaker position $\tilde{\ell}_{1}$ are related.

3) The loudspeaker membrane position is controlled in finite time by the current $\tilde{i}_{1}$ in order to achieve the desired pressure $p_{\mathrm{c}}^{*}$ ensuring the pole placement of the upper membrane.

${ }^{5}$ In a practical implementation, $\tilde{z}_{1}$ is deduced from a measurement of the amplitude of the upper membrane, obtained through a laser or piezoelectric sensor. 


\section{B. Control of the membrane by pole placement}

The first two equations of the PHS (13) represent the vibration of the first axisymmetric mode of the circular membrane subjected to a distributed pressure $\tilde{p}_{\mathrm{c}}\left(\triangleq p_{\mathrm{c}} / p_{0}=\right.$ $-\gamma \tilde{V}_{\mathrm{c}}$ ). If we are able to control this pressure by a pole placement, i.e.,

$$
\tilde{p}_{\mathrm{c}}^{*}(\tilde{t})=-k_{1} \tilde{z}_{1}(\tilde{t})-k_{2} \partial_{\tilde{t}} \tilde{z}_{1}(\tilde{t}),
$$

with $k_{1}$ and $k_{2}$ parameters that have been chosen accordingly, we obtain a desired frequency and damping of the first mode. This is possible because the subsystem of the first mode of the membrane controlled by the pressure satisfies the Kalman controllability condition (see e.g. [27]).

\section{Coupling between the three subsystems}

The third equation of the PHS (13) provides a relation between the temporal evolution $\tilde{z}_{1}$ of the first membrane mode, the pressure in the cavity $\tilde{p}_{\mathrm{c}}$ and the loudspeaker position $\tilde{\ell}_{1}$. It corresponds to

$$
\frac{1}{\gamma} \tilde{p}_{\mathrm{c}}(\tilde{t})=\frac{\sqrt{\pi} \alpha \kappa}{B \chi_{1}} \tilde{\lambda}_{1} \operatorname{sign}\left(J_{0}\left(\tilde{\lambda}_{1}\right)\right) \tilde{z}_{1}(\tilde{t})+\frac{\beta_{1}}{\beta_{2}} \tilde{\ell}_{1}(\tilde{t}) .
$$

If we substitute an amplitude measurement $\tilde{z}_{1}^{\mathrm{m}}(\tilde{t})$ and a pressure measurement $\tilde{p}_{\mathrm{c}}^{\mathrm{m}}(\tilde{t})$ in this unimodal relation, we obtain an (implicit) measurement for the loudspeaker position $\tilde{\ell}_{1}^{\mathrm{m}}(\tilde{t})$. If we use a pressure reference $\tilde{p}_{\mathrm{c}}^{*}(\tilde{t})$ corresponding to the pole placement (14) instead, we obtain a reference for the loudspeaker position $\ell_{1}^{*}(\tilde{t})$.

\section{Finite-time control of the loudspeaker}

We recall some elements before stating the obtained control law.

1) Definition and finite-time control of a double integrator: A system controlled in finite time will reach an equilibrium point in a finite time. A more formal definition of finite-time stability is given below.

Definition 3 (Finite-time stability [15]): Let $\dot{\boldsymbol{x}}=\boldsymbol{F}(\boldsymbol{x})$ represent a closed-loop system, with $\boldsymbol{F}(\boldsymbol{x})$ continuous and $\boldsymbol{F}(\mathbf{0})=0$. Let $\boldsymbol{\Phi}^{t}\left(\boldsymbol{x}_{0}\right)$ be the time evolution of the state for a given initial state $\boldsymbol{x}_{0}$.

The origin is a finite-time stable equilibrium if there exists an open neighborhood $U \subset \mathbb{R}^{n}$ of the origin, where following statements hold:

1) Finite-time convergence. There exists a settling-time function $T: U \backslash\{0\} \rightarrow \mathbb{R}_{\geq 0}$ such that for each $\boldsymbol{x}_{0} \in$ $U \backslash\{0\}$, the evolution $\boldsymbol{\Phi}^{t}\left(\boldsymbol{x}_{0}\right)$ is defined and unique on $t \in\left[0, T\left(\boldsymbol{x}_{0}\right)\left[\right.\right.$ and $\lim _{t \rightarrow T\left(\boldsymbol{x}_{0}\right)} \boldsymbol{\Phi}^{t}\left(\boldsymbol{x}_{0}\right)=0$.

2) Lyapunov stability. There exists a monotonically increasing function $\delta(\cdot), \delta(0)=0$, such that for each $\boldsymbol{x}_{0} \in U,\left\|\boldsymbol{\Phi}^{t}\left(\boldsymbol{x}_{0}\right)\right\| \leq \delta\left(\left\|\boldsymbol{x}_{0}\right\|\right)$ for each $t \geq 0$.

Furthermore, if $U=\mathbb{R}^{n}$, the origin is globally finite-time stable.

Lemma 1 (Finite-time control of a double integrator [16]): Consider the double integrator $\dot{z}_{1}=z_{2}, \dot{z}_{2}=v$. The origin is a finite-time stable equilibrium point of this system when it is controlled by the input $v=-k_{1}\left\lfloor z_{1}\right\rceil^{\frac{\xi}{2-\xi}}-k_{2}\left\lfloor z_{2}\right\rceil^{\xi}$, with $\left.k_{1}, k_{2}>0, \xi \in\right] 0,1\left[\right.$ and $\lfloor x\rceil^{\xi} \triangleq \operatorname{sgn}(x)|x|^{\xi}$.
2) Finite-time control law for the loudspeaker current: The finite-time control law of Lemma 1 was applied to a mass-spring-damper system in [28]. A passive version of the controller was proposed, in the sense that it can be reinterpreted as a PHS and as such provides robustness against bad parameter estimation. In [29], these considerations were used to formulate a passive law that controls the position $\tilde{\ell}_{1}(\tilde{t})$ of a loudspeaker to a reference trajectory $\tilde{\ell}_{1}^{*}(\tilde{t})$ in finite time.

The last equation of the model (13) represents the (dimensionless) loudspeaker ODE (5) and can be rewritten as

$$
\frac{1}{\beta_{2}} \partial_{\tilde{t}}^{2} \tilde{\ell}_{1}+\frac{\zeta}{\beta_{2}} \partial_{\tilde{t}} \tilde{\ell}_{1}+\beta_{2} \tilde{\ell}_{1}=-\frac{\beta_{1}}{\gamma} \tilde{p}_{\mathrm{c}}+\tilde{i}_{1} .
$$

By identification, one finds that the corresponding finite-time passive law is

$$
\begin{array}{r}
\tilde{i}_{1}=\frac{\beta_{1}}{\gamma} \tilde{p}_{\mathrm{c}}^{\mathrm{m}}+\beta_{2} \tilde{\ell}_{1}^{\mathrm{m}}+\frac{\zeta}{\beta_{2}} \partial_{\tilde{t}} \tilde{\ell}_{1}^{\mathrm{m}}-\bar{k}_{3}\left(\tilde{\ell}_{1}^{\mathrm{m}}-\tilde{\ell}_{1}^{*}\right)-\bar{k}_{4}\left(\partial_{\tilde{t}} \tilde{\ell}_{1}^{\mathrm{m}}-\partial_{\tilde{t}} \tilde{\ell}_{1}^{*}\right) \\
-\frac{1}{\beta_{2}}\left(\bar{k}_{1}\left[\tilde{\ell}_{1}^{\mathrm{m}}-\tilde{\ell}_{1}^{*}\right]^{\frac{\bar{\alpha}}{2-\bar{\alpha}}}+\bar{k}_{2}\left\lfloor\partial_{\tilde{t}} \tilde{\ell}_{1}^{\mathrm{m}}-\left.\partial_{\tilde{t}} \tilde{\ell}_{1}^{*}\right|^{\bar{\alpha}}\right), \quad\right. \text { (16) }
\end{array}
$$

with control parameters $\left.\bar{k}_{1}, \bar{k}_{2}>0, \bar{\alpha} \in\right] 0,1\left[, \bar{k}_{3}>\beta_{2}\right.$, $\bar{k}_{4}>\frac{\zeta}{\beta_{2}}$. We substitute the signals $\tilde{\ell}_{1}^{m}$ and $\tilde{\ell}_{1}^{*}$ (and their time derivatives) as explained below Eq. (15).

Stability: The global system of the membrane and loudspeaker can be viewed in a singular perturbation way. More precisely, the membrane controlled by the pressure can be interpreted as a slow subsystem, exponentially stabilized, whereas the loudspeaker can be interpreted as a fast subsystem, controlled in finite-time through the current. The whole system is then exponentially stabilized according to Tikhonov's theorem (see e.g. [30, Thm. A.11]).

\section{SIMULATION}

\section{A. Energy-preserving discretization}

We simulate the controlled PHS model (13) using a method [31], [22] that ensures stability by preserving the power balance after time discretization by jointly replacing the time derivative of the state by a finite difference and the gradient of the Hamiltonian by a so-called discrete gradient.

\section{B. Simulation}

We simulate the unimodal PHS tom-tom model (13) for which the control law 116 is activated at $\tilde{t}=15$.

Model parameters: initial state $\left[\begin{array}{lllll}0 & -1 & 0 & 0 & 0\end{array}\right]^{\top}, B=$ 5.54, $\tilde{\lambda}_{1}=3.08, \kappa=0.171, \gamma=1.4, \alpha=1.33, \beta_{1}=$ $0.768, \beta_{2}=0.353, \zeta=.0923, \xi=0.1$. Control parameters: $k_{1}=0.1, k_{2}=0.0005, \bar{\alpha}=0.8, \bar{k}_{1}=10, \bar{k}_{2}=10, \bar{k}_{3}=$ $2 \beta_{2}, \bar{k}_{4}=2 \zeta / \beta_{2}$. Simulation parameter: $\delta \tilde{t}=0.01$.

The resulting time evolution of the state is shown in Figure 2 and the periodogram of $\tilde{z}_{1}$ for the (un)controlled cases is shown in Figure 3 . The simulation confirms that the controller is able to modify the frequency of $\tilde{z}_{1}$.

Note that the membrane movement exhibits a second, lower frequency in the non-controlled case, due to the coupling with the cavity.

\footnotetext{
${ }^{6}$ Note that the practical implementation of this control law based on the measurement of $\tilde{p}_{\mathrm{c}}$ and $\tilde{z}_{1}$ involves additional numerical derivation.
} 


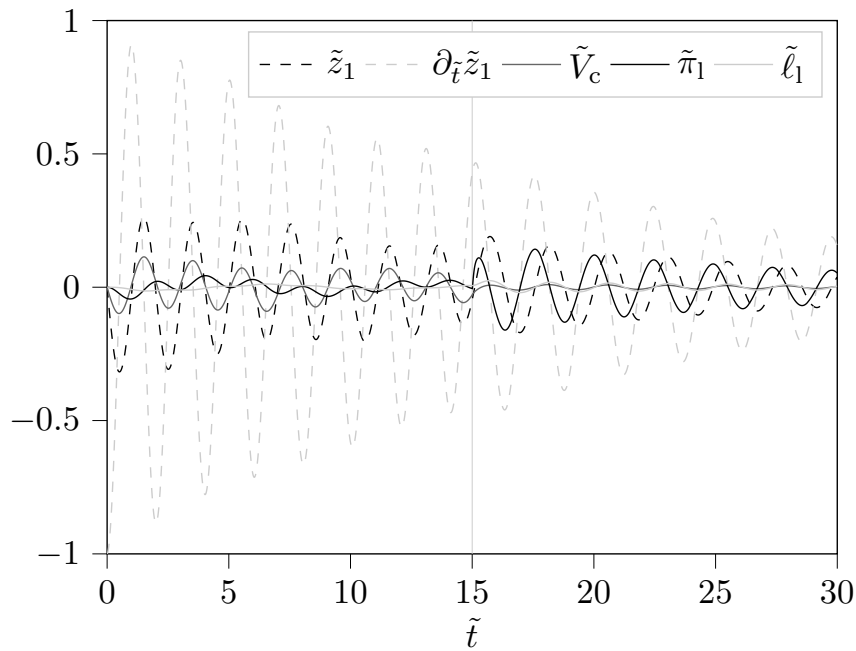

Fig. 2. Simulation of the unimodal tom-tom model: time evolution of the state

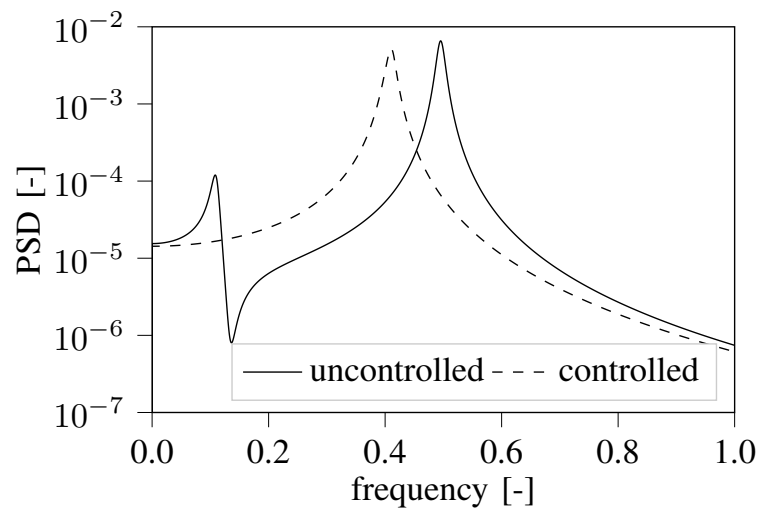

Fig. 3. Simulation of the unimodal tom-tom model: periodogram of $\tilde{z}_{1}$

\section{CONCLUSION AND FUTURE WORKS}

We have designed a controller for a unimodal model of the membrane. The next step is to extend this result to the multimodal case, considering spill-over, mode interaction and stability of the controller. Furthermore, we wish to implement the controller on a testbench that is under construction.

\section{REFERENCES}

[1] S. J. Elliott and P. A. Nelson, "Active noise control," IEEE signal processing magazine, vol. 10, no. 4, pp. 12-35, 1993.

[2] C. C. Fuller, S. Elliott, and P. A. Nelson, Active control of vibration. Academic Press, 1996.

[3] B. d'Andréa-Novel, B. Fabre, and J.-M. Coron, "An acoustic model for automatic control of a slide flute," Acta Acustica united with Acustica, vol. 96 , no. 4 , pp. 713-721, 2010.

[4] H. Boutin, "Méthodes de contrôle actif d'instruments de musique. cas de la lame de xylophone et du violon." Ph.D. dissertation, Université Pierre et Marie Curie - Paris VI, 2011.

[5] S. Benacchio, "Contrôle actif modal appliqué aux instruments de musique à cordes," Ph.D. dissertation, Université Pierre et Marie Curie - Paris VI, 2014.

[6] T. Meurisse, "Contrôle actif appliqué aux instruments de musique à vent," Ph.D. dissertation, Université Pierre et Marie Curie - Paris VI, 2014.
[7] M. Jossic, A. Mamou-Mani, B. Chomette, D. Roze, F. Ollivier, and C. Josserand, "Modal active control of Chinese gongs," The Journal of the Acoustical Society of America, vol. 141, no. 6, pp. 4567-4578, 2017.

[8] R. Worland, "Normal modes of a circular drumhead under nonuniform tension," The Journal of the Acoustical Society of America, vol. 127 , no. 1, pp. 525-533, 2009.

[9] G. Sathej and R. Adhikari, "The eigenspectra of Indian musical drums," The Journal of the Acoustical Society of America, vol. 125, no. 2, pp. 831-838, 2009.

[10] S. Tiwari and A. Gupta, "Effects of air loading on the acoustics of an Indian musical drum," The Journal of the Acoustical Society of America, vol. 141, no. 4, pp. 2611-2621, 2017.

[11] R. S. Christian, R. E. Davis, A. Tubis, C. A. Anderson, R. I. Mills, and T. D. Rossing, "Effects of air loading on timpani membrane vibrations," The Journal of the Acoustical Society of America, vol. 76, no. 5, pp. 1336-1345, 1984

[12] L. Rhaouti, A. Chaigne, and P. Joly, "Time-domain modeling and numerical simulation of a kettledrum," The Journal of the Acoustical Society of America, vol. 105, no. 6, pp. 3545-3562, 1999.

[13] M. Krstic and A. Smyshlyaev, Boundary control of PDEs: A course on backstepping designs. Siam, 2008, vol. 16.

[14] V. T. Haimo, "Finite time controllers," SIAM Journal on Control and Optimization, vol. 24, no. 4, pp. 760-770, 1986.

[15] S. P. Bhat and D. S. Bernstein, "Finite-time stability of continuous autonomous systems," SIAM Journal on Control and Optimization, vol. 38 , no. 3, pp. 751-766, 2000.

[16] E. Bernuau, W. Perruquetti, D. Efimov, and E. Moulay, "Robust finite-time output feedback stabilisation of the double integrator," International Journal of Control, vol. 88, no. 3, pp. 451-460, 2015.

[17] L. E. Kinsler, A. R. Frey, A. B. Coppens, and J. V. Sanders, "Fundamentals of acoustics," Fundamentals of Acoustics, 4th Edition, pp. 560. ISBN 0-471-84789-5. Wiley-VCH, December 1999., p. 560, 1999.

[18] K. F. Graff, Wave motion in elastic solids. Courier Corporation, 2012.

[19] P. M. Morse, Vibration and sound. American Society of Acoustics, 1995.

[20] B. M. Maschke and A. J. van der Schaft, "Port-controlled Hamiltonian systems: modelling origins and systemtheoretic properties," IFAC Proceedings Volumes, vol. 25, no. 13, pp. 359-365, 1992.

[21] A. van der Schaft, "Port-Hamiltonian systems: an introductory survey," in Proceedings of the international congress of mathematicians, vol. 3. Citeseer, 2006, pp. 1339-1365.

[22] A. Falaize and T. Hélie, "Passive guaranteed simulation of analog audio circuits: A port-Hamiltonian approach," Applied Sciences, vol. 6, no. 10 , p. $273,2016$.

[23] R. Ortega, A. van der Schaft, B. Maschke, and G. Escobar, "Interconnection and damping assignment passivity-based control of portcontrolled Hamiltonian systems," Automatica, vol. 38, no. 4, pp. 585596, 2002.

[24] R. F. Curtain and H. Zwart, An introduction to infinite-dimensional linear systems theory. Springer, 1995.

[25] T. Hélie and D. Matignon, "Nonlinear damping models for linear conservative mechanical systems with preserved eigenspaces: a portHamiltonian formulation," IFAC-PapersOnLine, vol. 48, no. 13, pp. 200-205, 2015

[26] J. A. Villegas, "A port-Hamiltonian approach to distributed parameter systems," Ph.D. dissertation, University of Twente, 2007.

[27] B. d'Andréa-Novel and M. De Lara, Control theory for engineers A primer. Springer, 2013.

[28] M. Wijnand, B. d'Andréa-Novel, T. Hélie, et D. Roze, "Contrôle des vibrations d'un oscillateur passif : stabilisation en temps fini et par remodelage d'énergie," in Congrès Français d'Acoustique, 2018.

[29] T. Lebrun, M. Wijnand, T. Hélie, D. Roze, and B. d'Andréa-Novel, "Electroacoustic absorbers based on finite-time control of loudspeakers: a numerical investigation," in International Nonlinear Dynamics Conference, 2019.

[30] C. C. de Wit, G. Bastin, and B. Siciliano, Theory of Robot Control. Springer-Verlag, 1996.

[31] Thomas Hélie, Antoine Falaize, et Nicolas Lopes, "Systèmes Hamiltoniens à Ports avec approche par composants pour la simulation à passivité garantie de problèmes conservatifs et dissipatifs," in Colloque National en Calcul des Structures, vol. 12, 2015. 